\title{
Subjective and objective evaluation of sense of space for vehicle occupants based on anthropometric data
}

\author{
Pitarn Hiamtoe ${ }^{\mathrm{a}, \mathrm{b}^{*}}$, Florian Steinhardt ${ }^{\mathrm{a}, \mathrm{b}}$, Uwe Köhler ${ }^{\mathrm{a}}$ and Klaus Bengler ${ }^{\mathrm{b}}$ \\ ${ }^{a}$ Department of Geometric Design and Integration Body, BMW AG, 80788 Munich, Germany \\ ${ }^{\mathrm{b}}$ Institue of Ergonomics, Technische Universität München, Boltzmannstr. 15, 85747 Garching, Germany
}

\begin{abstract}
At present, the number of the vehicle requirements has been continuously increasing. These requirements can be related to the customer as well as the technical requirements. Among these, the "feeling of space" of the occupants inside the vehicles can be regarded as one of the most important factors. In this respect, the driver and passengers should be able to experience positive feeling of space inside the vehicle. There are numerous factors that can influence the sense of space inside the vehicle. These include geometry (vehicle dimensions), light exposure, ambient lights, colors, material selection and material surface. Depending on the selection, the sense of space can be dramatically influenced by these factors. In general, human feeling is subjective and cannot be measured by any instrument. The measure can nevertheless be carried out by utilizing the method of subjective evaluation. Throughout the experiments, the method of evaluation is developed and the factors which can influence the interior feeling are analyzed. In this process, psychological perception, architectural aspects and anthropometry are considered and knowledge from the other domains is transferred in the form of a multidisciplinary approach. The experiments with an aim to evaluate the overall sense of space in the vehicle are carried out based on the physical mock up of BMW 1 series (E87). The space perception with different interior dimensions and anthropometric data of test persons are also analyzed. The use of Computer Aided Technology was shown by CATIA V5, PCMAN and RAMSIS. The results show a good correlation between the subjective evaluation and the geometric values.
\end{abstract}

Keywords: sense of space, effect of space, space perception, PCMAN, objective evaluation of space effects

\section{Introduction}

The research on the vehicle's interior design can be regarded as one of the most important stages in vehicle development process. Nowadays, we are spending a lot of time in the car and tend to spend more time in the future due to traffic jams, traveling, and other daily routine. While driving or taking a ride, we expect a good ambience, well-being and relaxing emotion. During the journey, drivers and passengers are supposed to have a good experience. At the same time, the negative consequences like those which can harm one's health, are to be avoided at all cost. Stress, pain, and discomfort are to be minimized. In this regard, we are to optimize various are in the interior to improve the room atmosphere and to increase the positive feeling. A study on one new car model in the luxury class has shown that increasing the dimension alone does not lead to the desired results. The effect of the interior on the occupants is a very complex issue with a number of related influencing factors. Apart from the vehicle dimension, a change in color, light exposure, material selection, surface material, design evaluation, and human factors can lead to a substantial effect on the occupants. To understand the problem better, the knowledge beside those attained from the engineering field, i.e. from the other branches like architecture, psychology, and psychophysics, is to be implemented in this study [4].

\footnotetext{
${ }^{*}$ Corresponding author. E-mail: hiamtoe@,lfe.mw.tum.de
} 
The main challenge is that affection is objective and cannot be measured by any devices. To tackle this difficulty, the occupants are to be used as a measure instrument of spatial properties (interior). Nevertheless, this evaluation approach might still poses some problems as shown listed below: (adapted from A. Neukum [7])

- How the interior attracted the occupants? (Question of the qualities of vehicle's interior attraction)

- How can the occupants perceive the difference of the car interior parts?

(Question of skill differentiation)

- Which relationships exist between the physical parameters and the evaluation? (Problem: methods of the "subjective versus the objective evaluation")

- Which effects due to various designs impact the feeling of safety and sense of space? (Demand of safety versus expectation of interior design)

\section{Methods}

\subsection{Definitions}

Since this paper is related to several branches, many terms should be defined in this section to make a common understanding before further reading. The term, "sense of space", is not new. Nevertheless, it can be difficult to define. We hear this word often from the architectural field but what does it mean in our understanding and what is actually the sense of space? Sense of space cannot be described through five human senses. Thus, it is not possible to define the sense of space with a certain definition. A person can evaluate this sense of space based on his common sense. The end result is either "like" or "dislike". However, this kind of sense is likely to be influenced by the surrounding, which can also affect this decision depends on the mood. These three terms below should be defined to ensure the common understanding throughout this paper.

Perception is a psycho-physical process, in which an organism develops an ostensive representation of its own body and environment according to internal and external stimuli. The field of philosophy deals with perception as one of the fundamental principles of human cognition. Perception psychology investigates the underlying information processing and neurophysiology investigates the underlying organic principles of perception $[2,6]$.

Effect of space can be defined as objective information to space, room or spatiality.

Sense of space is quite the contrary and can be defined as subjective interpretation and subjective evaluation of the perceptive of room effects.

These definitions are illustrated in Figure 1.

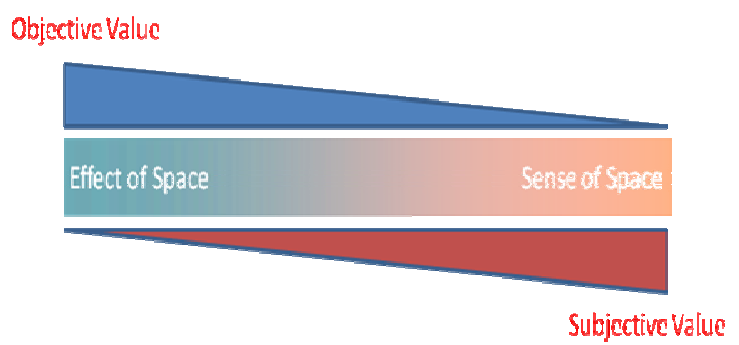

Fig. 1. Definition of subjective and objective value in terms of space perception

\subsection{General proceeding}

The general proceeding is shown in Figure 2. In the first step involves the preparation of the test object. In this case, we have the test object from BMW 1 series (E87). This prototype is used as a real car interior. Subjective analysis and objective analysis are consequently elaborated and tested. In the subjective evaluation is, for example, the space perception can be studied. Test persons can evaluate their perception and their interpretation in point system, or in words like too small, narrow, big, extravagant, etc. The information from this objective analysis is data that can be measured. If we focus on the evaluation of the sense of space under the influencing factors "geometry and car dimension", e.g. the distance of the Test person to the car parts like head clearance, shoulder clearance, leg room, the height of the door belt, the position of the pillar or the windshield et 


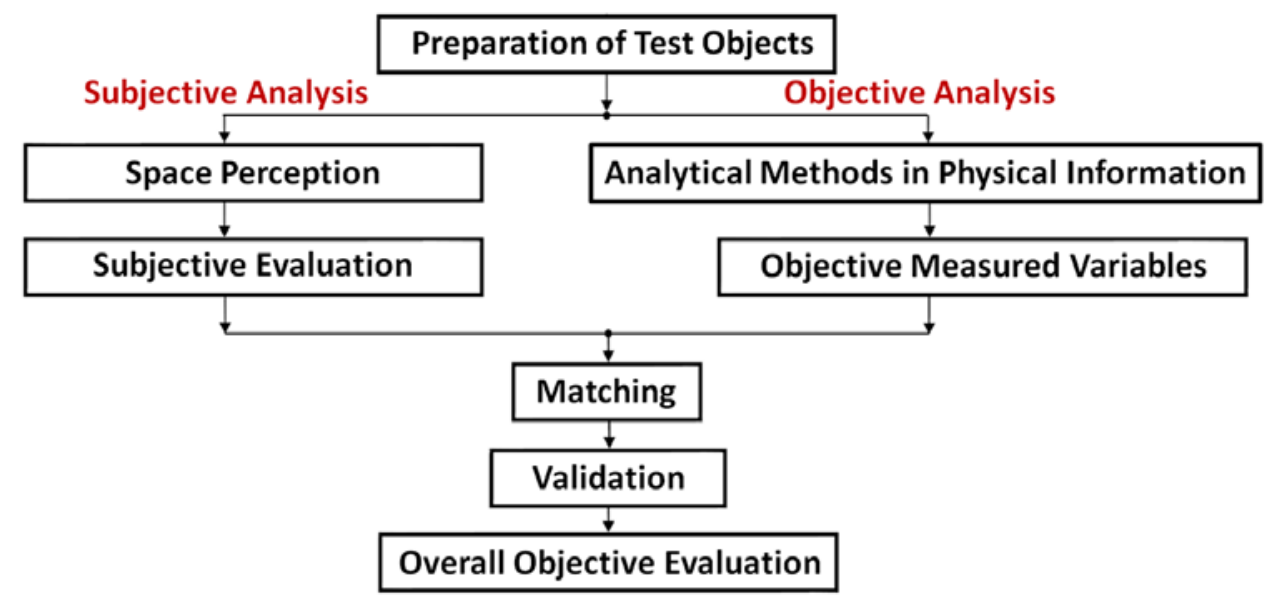

Fig. 2. General proceeding of the evaluation of the sense of space (adapted from Klaus Genuit [3], 2001)

\section{Experiment}

\subsection{Participants}

30 adult participants ( 8 women and 22 men) from the Technische Universität München (TUM) took part in this study. The average age of the participants is 30.27 years $(\mathrm{SD}=11.03$, range $18-62$ years $)$. The average height is $178.12 \mathrm{~cm}(\mathrm{SD}=11.8$, range 162 $196 \mathrm{~cm}$ ) with the average body weight of $72.17 \mathrm{~kg}$ $(\mathrm{SD}=12.99$, range $46-91 \mathrm{~kg})$. All participants have normal or corrected-to-normal vision.

Subjects had to evaluate the sense of space for the BMW 1 series mock up with 25 configurations. In this case, only the geometric factor (interior dimension) was considered. Perceived quality, color, contrast, design and features were not included during the evaluation in this study.

\subsection{Prototype system (Test object)}

The construction of the test object is based on the 1 series (E87). The reason is that, we would like to study and improve a sense of space in a small car. The building of the universal cockpit (test object in real system) is more complex than those in the virtual reality system. It should be noted that not all parameters can be varied within the cockpit prototype. In order to examine the effects of the space on the test persons as well as the corresponding space perception, the dimension and volume of the car interior are varied. In short, the space of interior can be increased and decreased. There are three parameters, which can be varied:

- Vertical translation of the roof panel along the $\mathrm{z}$-axis

- Translation of the door, a-, b-pillar and side panel along the $y$-axis

- The dimension of the steering-wheel

Test persons can evaluate the sense of space in this real cockpit under the variation of these three parameters. The implementation of the mock up from CAD-model to the real cockpit is shown in the Figure 3-5.

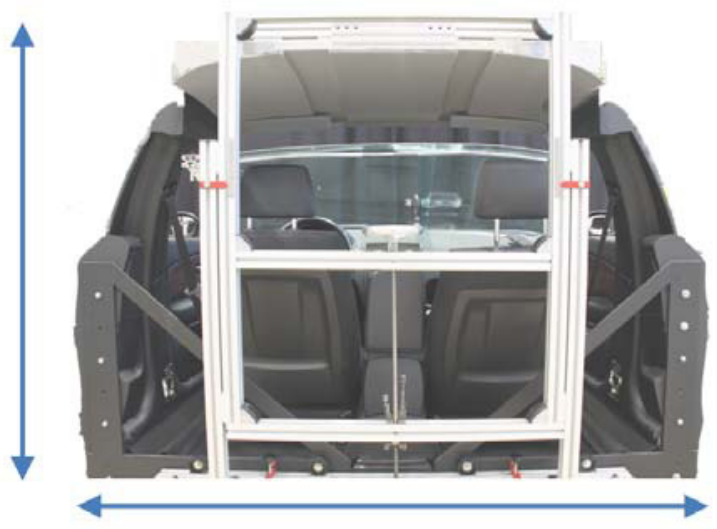

Fig. 3. Variation of the car height and car width 


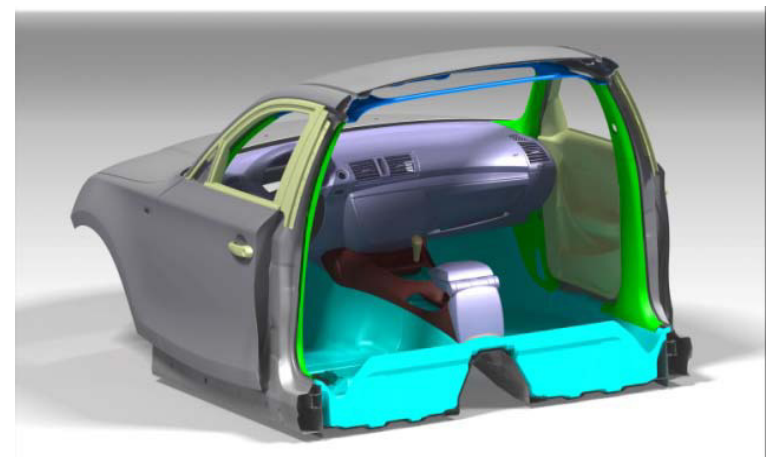

Fig. 4. Preparation of the mock up in CAD system (CATIA V5)

\subsection{Procedure}

The duration of the experiment is set to 60 minutes and separated in two parts. The first part is for anthropometric data acquisition and the second part is for the evaluation.

During the first step, the anthropometric data of the subject was recorded by using a camera. Pictures of the subject were taken in two postures (Standing posture and Pharaoh posture) and from two perspectives (front view and side view). The pictures with Bitmap (.bmp) format can be used in PCMAN to create an anthropometric correct three-dimensional man model of the subject. PCMAN is software that is used to adjust the three-dimensional man model RAMSIS to individual anthropometries of a subject and to measure subject posture and movement [5].

The subject is consequently asked to complete the questionnaire and give his personal data in PHP program to evaluate sense of space in the mock up for 25 configurations. The scorecard for this part of the experiment is saved as a Microsoft Excel file with macros and is designed to random the sequences of 25 configurations before starting the experiment. In the dialogue with the subjects, the assessments in terms of sense of space are documented for each configuration and stored in the Excel file. After the evaluation of the last configuration, the picture of the subject is taken in a driving position (from the front view and from the side view). The participation of the subject ended with the synchronization and calibration of the mock up-camera.

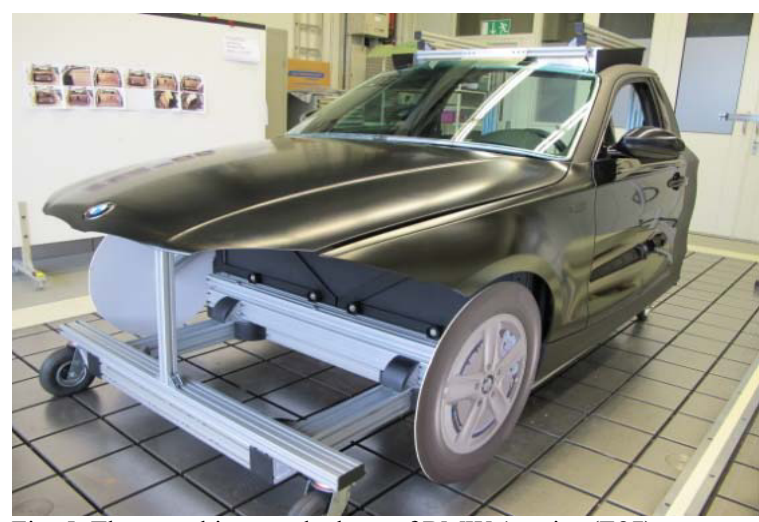

Fig. 5. The test object on the base of BMW 1 series (E87)

\section{Results}

The height and the width of the mock up for each configuration are shown in Table 1, which are sorted from number 1 to number 25 .

Table 1

The Dimension of each configuration

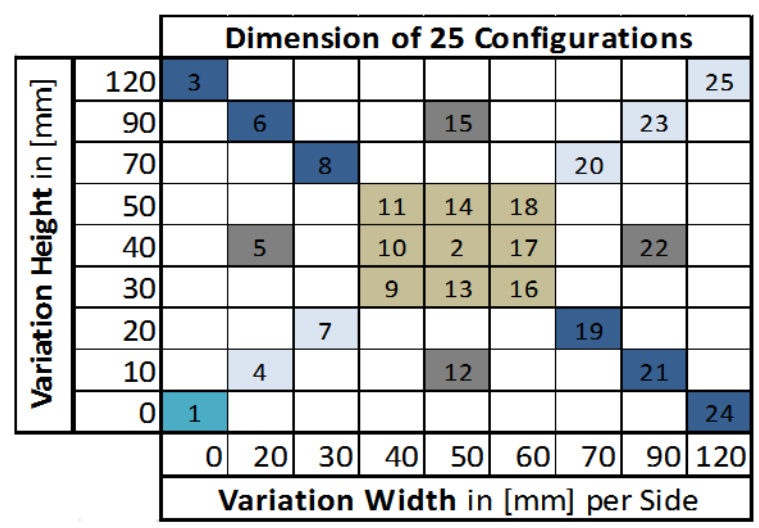

A tendency of the arithmetic mean of each evaluation is shown in Figure 6. In this histogram the sense of space is displayed in blue while the sense of safety is shown in red. To measure the variability of the mean values, the error bars are displayed in columns. The spacing between each different parts of the box helps indicate the degree of dispersion (spread), skewness in the data, and identify outliers [8]. These determine the $95 \%$ confidence interval. The confidence interval can be interpreted as an indication of the significance of the mean. The smaller volume of 
the area indicates more representative of the mean for the result.

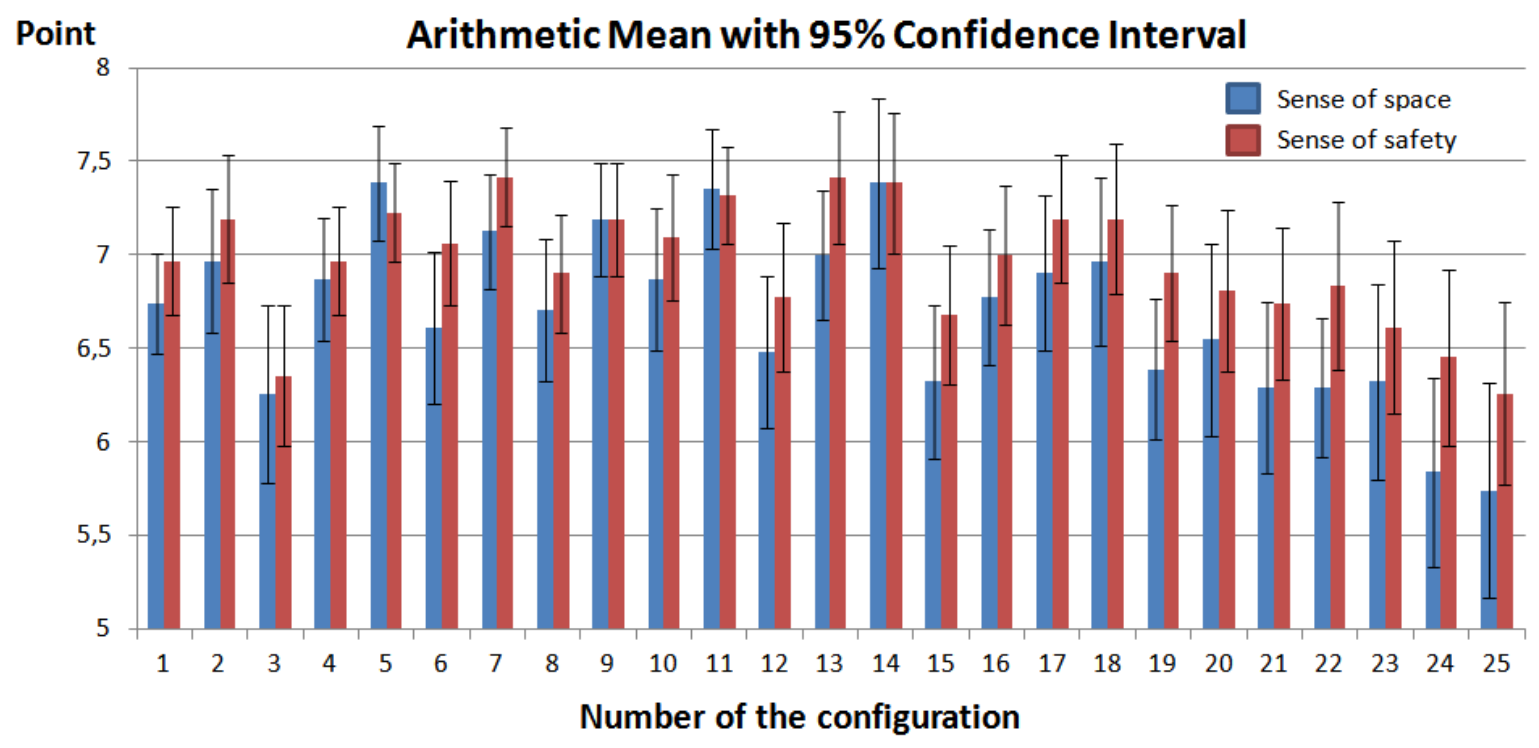

Fig. 6. Results of the evaluation sense of space and sense of safety for all configurations

\subsection{Regression analysis}

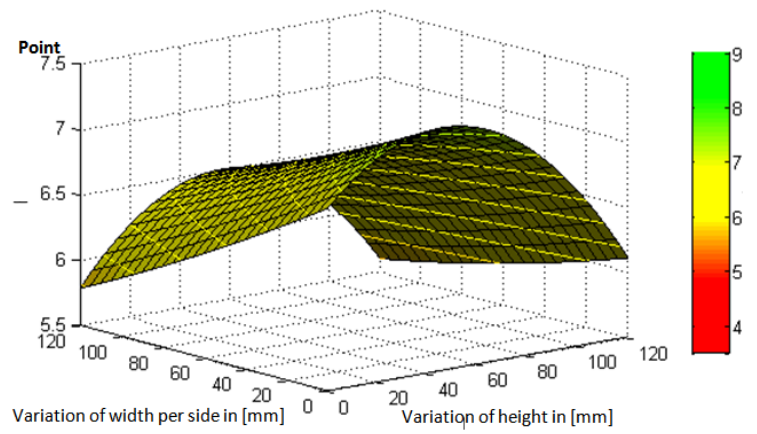

Fig. 7. Overall view of regression analysis for the sense of space

In Figure 7 the subjective evaluation is displayed as a function of door and roof position. It can be seen that the optimal sense of space can be reached with the roof height adjusted within the range of 40 and 50 $\mathrm{mm}$. In the E87 model, the starting width of the interior is set to $0 \mathrm{~mm}$. In this regard, the roof design for
E87 can be viewed as a crucial factor in which the sense of space of the passenger is hugely dependent upon. In comparison to the positional adjustment of the door, it can be noticed that the roof curvature of the vehicle possesses a greater impact on the sense of space of the passenger. The same trend has also been reported by Dainoff [1] and Tanoue [9] that the sense of space is largely influenced by the positioning of the deck surface and the roof of the vehicle respectively. 


\subsection{Influence of space perception}

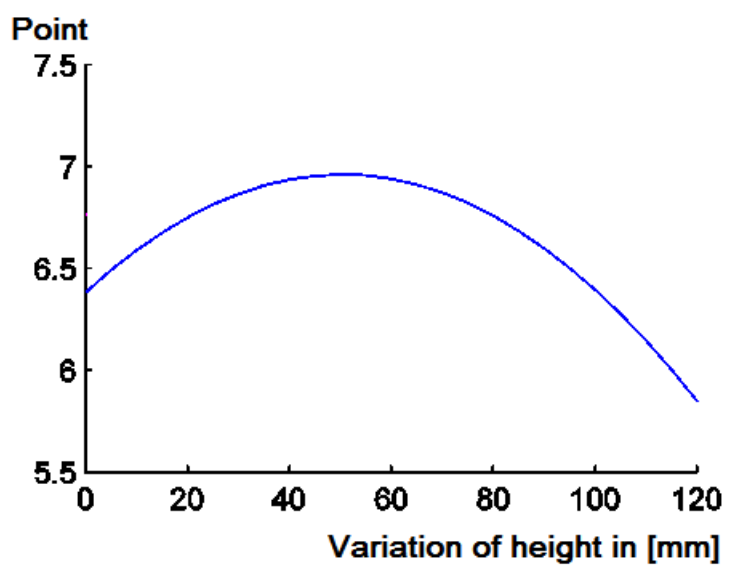

Fig. 8. Subjective evaluation of the sense of space according to the head clearance

In this study, some geometric quantities are also taken into consideration. These are, for instance, subjective evaluation of the sense of space as a function of the available head and shoulder room, overhead view and view obscurity. The selected results attained from this study are depicted in Figure 8. The influence of the roof and the edge of the roof can be represented as a function of the head room along the vertical direction. The curve evaluating the sense of space rises to attain the optimal value at the roof height equal to $50 \mathrm{~mm}$. Increasing the height further results in the curve deviating from this optimal value. Apparently, a passenger would feel uncomfortable when subjected to limited head room. At the same time, a large gap between the top of the head rest and the roof makes the passenger feel uneasy, thus worsens the feeling of space. Evaluating the entire adjustment range, it is shown that the optimal roof setting of 50 $\mathrm{mm}$ can be regarded as the ideal distance to attain the optimal sense of space as well as the sense of security.

\section{Conclusions}

The subjective and objective evaluations of the sense of space are becoming an important topic for automotive research and development to help resolve system designs. In this paper, the methods used to evaluate of the sense of space assisted by the use of computer aided technologies is presented. This method results in a reduction of time and cost required during the experiment. In this study, the important parameter of car interior which can influence the sense of space is determined. Furthermore, it was found that the human body size has a direct influence on the final result. For this reason the body measurement is required to be able to determine the space between the test person and the test object. These objective data were used to compare with the subjective evaluation. The methods of subjective and objective evaluation of space can be adapted to determine more test parameters. This is not only limited to the size of the car interior but for the other parameters like light and materials as well. In future work, conjoint analysis can be used to determine the optimal size of interior elements such as the dimension of Apillar trim or the height of door. These optimal sizes depend on the feeling of safety and the sense of space of the test subject. By utilizing the conjoint analysis, the effect of size depending on dark color and bright color can be found to further analyze the space perception of human under the influence of light and color [4].

\section{References}

[1] M.J. Dainoff, R.C. Sherman, D. Miskie and J. Grovesnor, Perceived Enclosedness of Schematic Architectural Space. In: Journal of Experimental Psychology: Human Perception and Performance. 1981, pp. 1349-1356

[2] J. Eichhorn, Design Parameter Shift Evaluation, Doctoral Thesis, Technische Universiteit Eindhoven, 2008, p. 25,

[3] K. Genuit, Parameter bei der Beurteilung von Fahreindrücken: Hören-Fühlen-Sehen-Wissen, Expert Verlag, 2001, pp. 143162

[4] P. Hiamtoe, U. Koehler and K. Bengler, Subjective and Objective Evaluation of the Sense of Space for Vehicle Occupants, 3rd FISITA World Automotive Congress 2010, Budapest, Hungary, No.F2010B061

[5] Institute of ergonomics, Technische Universität München, PCMAN User Handbook, 2007

[6] Meyers Lexikonverlag (2007), "Wahrnehmung", Retrieved September, 2007

[7] A. Neukum, Prof. H.P. Krüger and J. Schuller, Der Fahrer als Messinstrument für fahrdynamische Eigenschaften?, VDIBerichte Der Fahrer im 21. Jahrhundert, 2001, No. 1613, pp. 14-15

[8] D. Pettersen, Data Analysis and Statistics - boxplot, Scilab.org

[9] C. Tanoue, K. Ishizaka and M. Nagamashi, Kansei Engineering: A study on perception of vehicle interior image. In: International Journal of Industrial Ergonomics. 1997, pp. 115-128 